\title{
AN ITERATIVE METHOD FOR THE NUMERICAL INVERSION OF LAPLACE TRANSFORMS
}

\author{
CRISTINA CUNHA AND FERMIN VILOCHE
}

\begin{abstract}
We present an algorithm for the numerical inversion of Laplace transforms that is a particular case of the iterated regularization method proposed by Vainikko in 1982. To construct the finite-dimensional space, we use Laguerre polynomials. Error bounds for the approximations are derived.
\end{abstract}

\section{INTRODUCTION}

Let $X=L_{w}^{2}\left(R^{+}\right)$be the weighted Lebesgue space associated with $w(t)=$ $e^{-t}, Y=L^{2}([c, d]), d>c>0$ and $A: X \rightarrow Y$ the Laplace transform operator,

$$
(A x)(s)=\int_{0}^{\infty} e^{-s t} x(t) d t=y(s) .
$$

As is known, the problem of solving (1), for a given $y \in Y$, is ill-posed. The problem of determining $A^{+} y$, where $A^{+}$is the generalized inverse of $A$, is still ill-posed: the solution depends discontinuously upon $y$.

If we only know the perturbed data $y_{\delta}$, with

$$
\left\|y-y_{\delta}\right\|_{Y}<\delta,
$$

then one must use "regularization methods". This is a family of operators $R_{N}$ : $Y \rightarrow X$, indexed by some regularization parameters $N$, together with some strategy to choose the parameter such that $R_{N} y_{\delta}$ is an approximation to $A^{+} y$. There are also other kinds of perturbations when, instead of the operator $A$, we use an approximation $A_{N}$ such that $\left\|A_{N}-A\right\| \leq \beta_{N}$.

In this paper, we use the arguments presented by Vainikko, in [6], to design an algorithm for the inversion of the Laplace transforms of data with noise. The Laplace transform methods are helpful techniques for differential and integral equations; however when discretization is required to solve the problem in the Laplace domain, errors are introduced. Similar situations arise when we deal with the Laplace inversion of scientific measurements or observations.

\section{The Laguerre approximations}

If the data are only imprecisely known, that is, only $y_{\delta} \in Y$ is available satisfying (2), we can use the implicit successive approximation method [4]

Received by the editor January 22, 1992 and, in revised form, March 18, 1993.

1991 Mathematics Subject Classification. Primary 65R30, 65R10; Secondary 42C10.

(c)1995 American Mathematical Society 


$$
x^{k}=\left(\lambda I+A^{*} A\right)^{-1}\left(\lambda x^{k-1}+A^{*} y_{\delta}\right), \quad \lambda>0,
$$

where $A^{*}$ is the adjoint operator of $A$.

Let $V_{1} \subseteq V_{2} \subseteq \cdots$ be a sequence of finite-dimensional subspaces of $X$ where $V_{N}$ is spanned by the Laguerre polynomials of degree $\leq N$ [1]. The Laguerre polynomials, $\phi_{i}(t)$, are such that

$$
\int_{0}^{\infty} e^{-t} \phi_{i}(t) \phi_{j}(t) d t=\delta_{i j}
$$

and they form a complete set in $L_{w}^{2}\left(R^{+}\right)$[2, for example]. We will denote by $P_{N}$ the orthogonal projection of $X$ onto $V_{N}$ and $A_{N}=A P_{N}$.

In the finite-dimensional subspace $V_{N}$, we define the approximation

$$
x_{N}=\sum_{i=1}^{N} a_{i} \phi_{i}(t)
$$

such that

$$
\left\langle\left(\lambda I+A_{N}^{*} A_{N}\right) x_{N}^{k+1}, \phi_{j}\right\rangle=\left\langle\lambda x_{N}^{k}+A_{N}^{*} y_{\delta}, \phi_{j}\right\rangle, \quad j=0, \ldots, N, \lambda>0,
$$

where $\langle\cdot, \cdot\rangle$ is the usual inner product in $X$.

Let $\psi_{i}(s) \in Y$ be the Laplace transform of $\phi_{i}(t)$. As we know,

$$
\phi_{i}(t)=\sum_{k=0}^{i}\left(\begin{array}{l}
i \\
k
\end{array}\right) \frac{(-t)^{k}}{k !}
$$

so that

$$
\psi_{i}(s)=\int_{0}^{\infty} e^{-s t} \phi_{i}(t) d t=\sum_{k=0}^{i}\left(\begin{array}{c}
i \\
k
\end{array}\right) \frac{(-1)^{k}}{s^{k+1}}=\frac{1}{s}\left(1-\frac{1}{s}\right)^{i} .
$$

With these functions we construct a matrix $\mathbf{M}$,

$$
M_{i j}=\int_{c}^{d} \psi_{i}(s) \psi_{j}(s) d s=\int_{c}^{d} \frac{1}{s^{2}}\left(1-\frac{1}{s}\right)^{i+j} d s=\frac{\tilde{c}^{i+j+1}-\tilde{d}^{i+j+1}}{i+j+1},
$$

where $\tilde{c}=(1-c) / c$ and $\tilde{d}=(1-d) / d$. If we define a vector $\mathbf{f}$,

$$
f_{i}=\int_{c}^{d} y_{\delta}(s) \psi_{i}(s) d s,
$$

the variational formulation of the implicit scheme (3), in $V_{N}$, will be

$$
(\lambda \mathbf{I}+\mathbf{M}) \mathbf{a}^{k}=\lambda \mathbf{a}^{k-1}+\mathbf{f} .
$$

For a given $\lambda>0$, we can state the Procedure

1. Do the Cholesky decomposition $\mathbf{L L}^{T}=\mathbf{M}+\lambda \mathbf{I}$;

2. $\mathbf{a}^{\circ}=0$

solve the system $\mathbf{L L}^{T} \mathbf{a}^{k}=\lambda \mathbf{a}^{k-1}+\mathbf{f}, k=1,2, \ldots$

We must observe that, in this process, the regularization is an important feature. The condition number of $\mathbf{M}$ becomes insupportable as $N$ increases; for example, if $N=15$, the condition number of $\mathbf{M}$ is $O\left(10^{19}\right)$.

By direct calculations we can show that the adjoint operator $A^{*}$ is, in this case,

$$
\left(A^{*} v\right)(t)=e^{t} \int_{c}^{d} e^{-t s} v(s) d s .
$$


Under the limitation $c>\frac{1}{2}$ it can be shown that $A^{*} v \in L_{w}^{2}\left(R^{+}\right)$. Also, we can see that $z(t)=\left(A^{*} v\right)(t), v(s) \in Y$, is an analytical function, and for $k=0,1, \ldots$

$$
z^{(k)}(t)=\int_{c}^{d} e^{-t(s-1)}(1-s)^{k} v(s) d s
$$

\section{ERROR BOUND ESTIMATES}

Assume that the data are on the interval $(c, d)$, with $c>\frac{1}{2}$, and, as before, let $\tilde{c}=(1-c) / c$ and $\tilde{d}=(1-d) / d$.

Lemma. Let $c>\frac{1}{2}$ and $\alpha=\max \{|\tilde{c}|,|\tilde{d}|\}$; then $\alpha<1$ and

$$
\beta_{N}=\left\|A-A_{N}\right\| \leq \sqrt{2} \frac{\alpha^{(N+1)}}{\left(1-\alpha^{2}\right)^{1 / 2}} .
$$

Proof. We know that

$$
\left\|A-A_{N}\right\|=\left\|A\left(I-P_{N}\right)\right\|=\left\|\left(I-P_{N}\right) A^{*}\right\|=\sup _{\|v\|=1}\left\{\left\|\left(I-P_{N}\right) A^{*} v\right\|_{X}\right\} .
$$

Let $z(t)=\left(A^{*} v\right)(t), v \in Y$, such that $\|v\|_{Y}=1$. Then

$$
\left\|\left(I-P_{N}\right) A^{*} v\right\|_{X}=\left\|\left(I-P_{N}\right) z(t)\right\|_{X}=\left\|z(t)-\sum_{i=1}^{N} b_{i} \phi_{i}(t)\right\|_{X},
$$

where $b_{i}$ are the Laguerre-Fourier coefficients of $z(t)$. The next step is to calculate the rate of convergence of the Laguerre-Fourier approximants. We will use a basic property of the Laguerre polynomials [1]:

$$
e^{-t} \phi_{k}(t)=\frac{1}{k !} \frac{d^{k}}{d t^{k}}\left(t^{k} e^{-t}\right), \quad k=0,1, \ldots
$$

By successive integration by parts, and the last equation, we get

$$
b_{k}=\int_{0}^{\infty} e^{-t} z(t) \phi_{k}(t) d t=\frac{(-1)^{k}}{k !} \int_{0}^{\infty} e^{-t} t^{k} z^{(k)}(t) d t .
$$

Using (5) and the Laplace transform of $t^{k}$, we obtain

$$
\begin{aligned}
b_{k} & =\frac{(-1)^{k}}{k !} \int_{c}^{d} v(s)(1-s)^{k} \int_{0}^{\infty} e^{-s t} t^{k} d t d s \\
& =(-1)^{k} \int_{c}^{d} \frac{1}{s}\left(\frac{1-s}{s}\right)^{k} v(s) d s .
\end{aligned}
$$

By the Schwarz inequality,

$$
\begin{aligned}
\left|b_{k}\right|^{2} & \leq\left\{\int_{c}^{d} \frac{1}{s^{2}}\left(\frac{1-s}{s}\right)^{2 k} d s\right\}\|v\|_{Y}^{2} \\
& =\left\{\frac{1}{2 k+1}\left(\tilde{c}^{2 k+1}-\tilde{d}^{2 k+1}\right)\right\}=[\gamma(k)]^{2 k}(\tilde{c}-\tilde{d}),
\end{aligned}
$$


and $\gamma(k) \in(\tilde{d}, \tilde{c})$ from the mean value theorem. But $-1<(1-x) / x<1$, if $x>\frac{1}{2}$, so that $-1<\tilde{d} \leq \gamma(k) \leq \tilde{c}<1$. If we choose $\alpha=\max \{|\tilde{c}|,|\tilde{d}|\}$, we have $\alpha<1$ and

$$
\sum_{k=N+1}^{\infty} b_{k}^{2} \leq(\tilde{c}-\tilde{d}) \sum_{i=N+1}^{\infty} \alpha^{2 i}=(\tilde{c}-\tilde{d}) \frac{\alpha^{2(N+1)}}{1-\alpha^{2}},
$$

and the lemma will follow.

The method of successive approximations (3) is familiar for ill-posed problems [3-6]. In particular, Theorem 1 in [6] is concerned with "a priori" specification of $k$. It claims that if

(i) $y \in R(A)$,

(ii) $x^{+} \in R\left(\left[A^{*} A\right]^{p / 2}\right)$, where $x^{+}$is the solution of (1) closest to 0 ,

(iii) $k=d_{1}\left(\delta+\beta_{N}\right)^{-2 /(p+1)}$ for some $d_{1}>0$,

then for any $\lambda>0$

$$
\left\|x^{k}-x^{+}\right\|_{X} \leq d_{2}\left(\delta+\beta_{N}\right)^{p /(p+1)}, \quad d_{2}=\operatorname{const}\left(p, d_{1}\right) .
$$

Our final conclusion follows directly from this result and the previous lemma:

Proposition. Under the conditions (i)-(iii), the successive approximations (4), with

$$
k=d_{1}\left(\delta+\frac{\alpha^{N+1}}{\left(1-\alpha^{2}\right)^{1 / 2}}\right)^{-2 /(p+1)},
$$

$d_{1}=$ constant and $\alpha$ defined in the previous lemma, will give $x_{k}$ such that

$$
\left\|x_{k}-x^{+}\right\|_{X} \leq d_{2}\left(\delta+\frac{\alpha^{N+1}}{\left(1-\alpha^{2}\right)^{1 / 2}}\right)^{p /(p+1)},
$$

where $d_{2}=d_{2}\left(p, d_{1}\right)$.

\section{NUMERICAL EXPERIMENTS AND CONCLUSIONS}

The examples of this section will give a qualitative idea of the performance of the proposed scheme. We choose $\lambda$ in such a way that the first iterate is an approximation for $x^{+}$; this is possible since the first iterate is the Tikhonov regularization solution. In this case there are "a priori" estimates for $\lambda$, as is shown in [3]. To stop the iterative process, we use a number of iterations $k$ such that

$$
\left\|A x_{N}^{k}-y_{\delta}\right\|<\mathrm{Tol}
$$

where Tol $=c_{1} \delta, c_{1}>0$ and $\delta$ from (2). The a posteriori stop rules are optimal but, in our case, they will demand excessive computational work.

In the numerical experiments we simulated the noise, taking $y_{\delta}(s)=y(s)+$ $\varepsilon \sin (100 s), \varepsilon>0$ and $s \in[1,5]$. The other parameters used in the examples are: $N=$ the maximal degree of the polynomials in $V_{N} ; k=$ the number of iterations required by the stopping criterion.

Example 1. If $y(s)=1 /(s+1.5)^{2}$, then $x(t)=t e^{-1.5 t}$. The noise on the data was simulated using $\varepsilon=10^{-2}$. The approximations were calculated taking $N=10$. In this example, $k=2$ iterations were required, i.e., $x_{10}^{2}(t)$ satisfies (10) with Tol $=1.4 \times 10^{-3}$. The comparison between $x(t)$ and $x_{10}^{2}(t)$ is shown in Figure 1. 


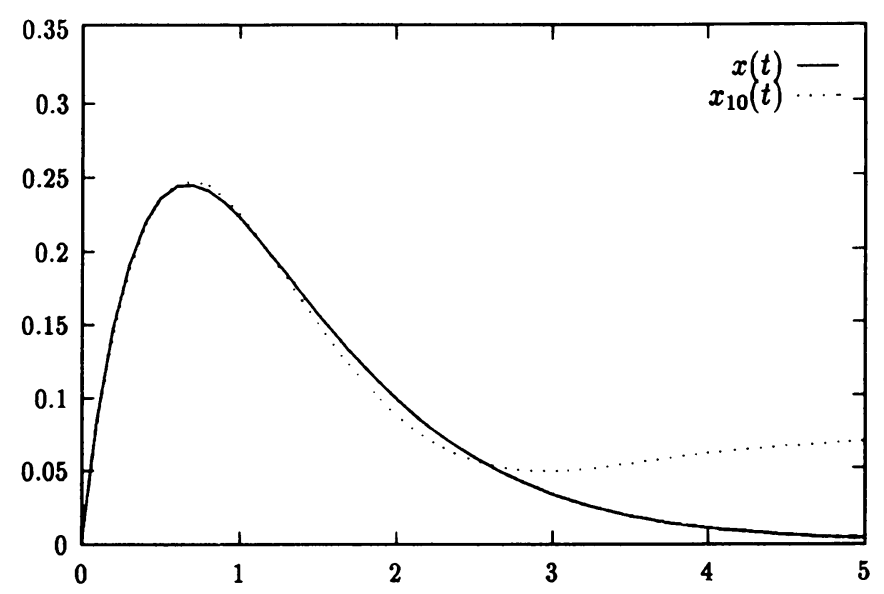

Figure 1. Example 1

Example 2. If $y(s)=\arctan \left(\frac{1}{s}\right)$, then $x(t)=\sin t / t$. In this case we tested $N=10$ and $N=15$, with noisy data corresponding to $\varepsilon=10^{-4}$. In the first case, $k=4$ iterations were performed to obtain (10) with a Tol $=1.4 \times 10^{-5}$. When $N=15, k=3$ iterations were required for a Tol $=1.4 \times 10^{-5}$. The results are shown in Figure 2.

The error bound presented here, as well as in the above numerical computations, encourage the use of the successive approximation method in the Laplace inversion problem. In different tests we got similar results, but especially good results were obtained when we used polynomials for $x(t)$.

The increase of the error for $t>5$, exhibited in the figures, is compatible with the norm used to measure the error: the weight $e^{-t}$ allows these large absolute errors. On the other hand, Laguerre polynomials exhibit strong oscillations when $N$ and $t$ increase [1]; we believe that this fact also produces damaging effects.

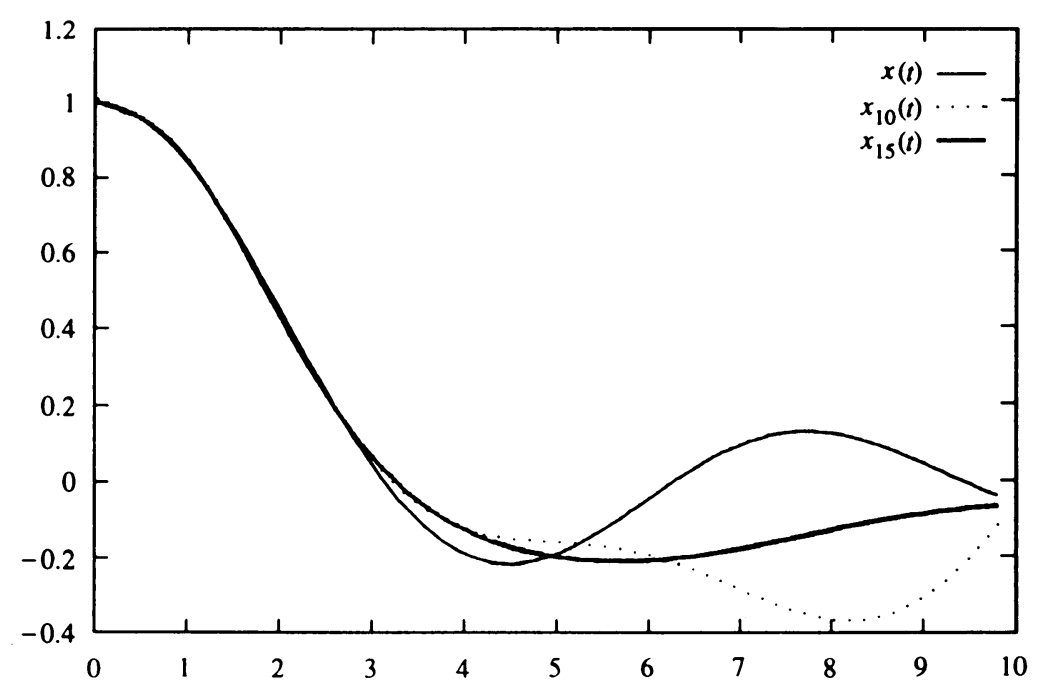

FIGURE 2. Example 2 


\section{BIBLIOGRAPHY}

1. A. Erdélyi, W. Magnus, F. Oberhettinger, and F. Tricomi, Higher transcendental functions, Vol. 2, McGraw-Hill, New York, 1953.

2. D. Gottlieb and S. Orszag, Numerical analysis of spectral methods, SIAM, Philadelphia, PA, 1977.

3. C. W. Groetsch, J. T. King, and D. Murio, Asymptotic analysis of a finite element method for Fredholm equations of the first kind, Treatment of Integral Equations by Numerical Methods (C. T. H. Baker and G. F. Miller, eds.), Academic Press, London, 1982, pp. 1-11.

4. J. T. King and D. Chillingworth, Approximation of generalized inverses by iterated regularization, Numer. Funct. Anal. Optim. 2 (1979), 449-513.

5. E. Schock, Comparison principles for iterative methods, Inverse and Ill-posed Problems (H. W. Engl and C. W. Groetsch, eds.), Academic Press, New York, 1987, pp. 185-193.

6. G. M. Vainikko, The discrepancy principle for a class of regularization methods, U.S.S.R. Comput. Math. and Math. Phys. 22 (1982), 1-19.

7. V. V. Vasin, Iterative methods for the approximate solution of ill-posed problems with a priori information and their applications, Inverse and Ill-posed Problems (H. W. Engl and C. W. Groetsch, eds.), Academic Press, New York, 1987, 211-229.

Departamento de Matemática Aplicada, IMECC-UniCAMP, C. P. 6065, 13081 Campinas-Sao Paulo, Brazil

E-mail address: cunha@ime.unicamp.br 